\title{
Role of Ultrasonography in Evaluation of Peripheral Nerves
}

\author{
Mandugula Aruna Devi' ${ }^{1}$, Ak Meena² \\ ${ }^{1}$ Department of Radiology, Nizam's Institute of Medical Sciences, Hyderabad, India \\ ${ }^{2}$ Department of Neurology, Nizam's Institute of Medical Sciences, Hyderabad, India \\ Email: arunabilu@yahoo.co.in,drmeenaak62@gmail.com
}

How to cite this paper: Aruna Devi, M. and Meena, A. (2018) Role of Ultrasonography in Evaluation of Peripheral Nerves. Open Journal of Internal Medicine, 8, 232-247.

https://doi.org/10.4236/ojim.2018.84022

Received: September 19, 2018

Accepted: December 18, 2018

Published: December 21, 2018

Copyright (c) 2018 by authors and Scientific Research Publishing Inc. This work is licensed under the Creative Commons Attribution International License (CC BY 4.0).

http://creativecommons.org/licenses/by/4.0/

\begin{abstract}
Ultrasonography is first line, cost-effective modality in imaging of peripheral nerves. High frequency probes allow high resolution imaging of peripheral nerves located at relatively superficial location. USG can diagnose and localize various pathologies that involve the peripheral nerves such as traumatic, inflammatory, infective, neoplastic, and traumatic neuropathies. USG exceptionally has an important role to play in evaluation of patients with suspected nerve injury, as MRI may not differentiate neural contusion from nerve disruption. Neurapraxic injury is seen as swollen nerve with hypoechoic appearance.
\end{abstract}

\section{Keywords}

HRUS, Superficial Peripheral Nerves, Nerve Lesions

\section{Introduction}

Peripheral nerves are affected by a number of disease processes like trauma, infection, inflammation, benign and malignant tumours, as well as entrapment neuropathies. Electrodiagnostic studies do not demonstrate morphologic information like site and degree of injury. Neurosonography provides a reliable diagnosis and localization in entrapment neuropathies, traumatic peripheral nerve injuries and tumours of the peripheral nerves [1] [2] [3], so it has become a useful supplementary tool for electrodiagnostic studies in these conditions. Characteristic nerve size changes in polyneuropathies have been reported as well. High resolution USG exceptionally has an important role to play in evaluation of patients with suspected nerve injury, as MRI may not differentiate neural contusion from nerve disruption. Neurapraxic injury is seen as swollen nerve with hypoechoic appearance. USG can map the nerve in its entire course. Compared to MRI 
USG with its high resolution is also cost-effective as in our country majority of population unable to afford costly diagnostic tests such as MRI [4] [5].

\section{Discussion}

\subsection{Technique}

A high frequency linear array probe $(6-13 \mathrm{MHz})$ is used [2]. Small foot print probe like hockey stick probe is used to image digital nerves. Focal zone and depth are optimally used to improve image quality. USG gel should be used liberally. The examination is started from a known anatomic landmark near the nerve [1] [3]. Once the nerve is localized in the short axis, it is traced cranially and caudally to check the size of that segment (as the various segments of each nerve differ in size) as shown in Table 1 and morphology (architecture). The probe is then turned in the long axis of the nerve and evaluated.

\subsection{Normal Appearances}

The normal nerve, in transverse section reveals small hypoechoic areas separated by hyperechoic septae giving a "honeycomb-like appearance". The septae represent interfascicular perineurium. The longitudinal sections also reveal the fascicular architecture, giving a "bundle of straws" appearance [2] [4]. The nerve is more echogenic compared to muscle which shows hypoechoic muscle fiber bundles with intervening echogenic perimysium. The tendon is more echogenic compared to nerve and shows a compact arrangement of echogenic fibrils [2] [4]. On dynamic examination, the nerves show sliding movement over the muscles and tendons. An altered movement or contour deformity during movement of the nerve gives us a clue to diagnose pathology.

Table 1. CSA $\left(\mathrm{mm}^{2}\right)$ values of various nerve segments in healthy subjects documented by Josef Bohm in his study on 56 healthy subjects [6].

\begin{tabular}{cc}
\hline Nerve & $10.0 \pm 2.9$ \\
C7 6 & $9.5 \pm 2.7$ \\
C5 & $5.6 \pm 1.6$ \\
Median arm & $8.9 \pm 1.8$ \\
Ulnar arm & $6.3 \pm 1.7$ \\
Radial arm & $4.2 \pm 1.0$ \\
Ulnar epicond & $7.6 \pm 2.1$ \\
Median forearm & $5.7 \pm 1.3$ \\
Ulnar forearm & $5.2 \pm 1.3$ \\
Spf radial forearm & $2.3 \pm 0.7$ \\
Median Carpal & $8.5 \pm 1.8$ \\
Peroneal & $8.9 \pm 2.0$ \\
Tibial & $9.6 \pm 2.2$ \\
Sural & $1.8 \pm 0.6$
\end{tabular}




\section{Ultrasound Appearances of Various Pathologies}

\subsection{Trauma}

Three peripheral nerve injury types are described based on the mechanism of injury, i.e. stretch injuries, laceration, and compression injuries [4]. Seddon defined 3 grades of nerve injury neurapraxia, axonotmesis, and neurotmesis based on the extent of injury to the three structural components of the peripheral nerve. Neurapraxia is injury with maintenance of nerve continuity. Axonotmesis is disruption of axons and myelin with intact epi and perineurium. Neurotmesis is complete disruption of the nerve [7]. Differentiate nerve injury in continuity from nerve transection is crucial element in treatment plan as neurapraxia and axonotmesis have good chance of recovery, while neurotmesis need surgery.

APPEARANCE with USG: USG demonstrates the site of injury, differentiate type of injury e.g.: nerve injury in continuity from nerve transection, detects foreign bodies, neuroma and scarring, in case with transection provides the distance between the stumps as is crucial in surgical plan. Neurapraxia is seen as swollen nerve with hypoechoic appearance which occur in case of compression and stretch injuries [Figure 1]. Complete and partial transaction of nerves seen as discontinuity in nerve with retraction of ends, which occur in laceration injuries. Stump or amputation neuromas as focal thickening or mass like lesions at the nerve ends is common association with transection injury [4].

\subsection{Tumors}

The most common nerve tumors are nerve sheath tumors which include schwannomas and neurofibromas. They are seen as well defined ovoid homogenous hypoechoic lesions with nerve entering and exiting from them. Accurate preoperative differential diagnosis of Schwannoma and other mass lesions is important because an incorrect preoperative diagnosis may lead to permanent neurologic deficits. Schwannomas arise from the nerve sheath, grow eccentrically, and have a capsule called the epineurium. As Schwannomas are well encapsulated so can be removed without disruption of the nerve. Neurofibroma, on the other hand, grow interstitially in the center of the nerve bundle within the endoneurium and are not encapsulated so require resection of the involved nerve segment. Differentiation between schwannomas and neurofibromas is known to be difficult on the basis of sonographic findings. Schwannoma has variable sonographic features including well defined margins, a homogenous hypoechoic appearance, a round to oval shape, through transmission and internal vascularity, visualization of the entering and exiting nerves, and the presence of a target sign. Certain predictors (lobulated contour, fusiform shape, and hypovascularity) could be helpful in differentiating between tumors. In addition, the nerve-tumor position, nerve tumor transition, and maximum to minimum diameter ratio are useful for distinguishing between schwannomas and neurofibromas. Schwannomas arise from the nerve sheath, grow eccentrically, and have a capsule called the epineurium, which results in a clearly defined nerve-tumor 
transition [Figure 2]. Neurofibroma, on the other hand, arise within the endoneurium and grow interstitially in the center of the nerve bundle and are not encapsulated. As a result, the nerve-tumor transition of neurofibromas is infiltrative [8] [9] [10] [Figure 3].

Morton's neuromas: Morton's neuromas are not true tumors. They are formed due to perineural fibrosis and thickening of plantar digital nerves. They occur due to chronic microtrauma. The usual location is in second or third intermetatarsal space at the level of heads of metatarsals. They are seen as hypoechoic mass, Scan in longitudinal axis demonstrates the plantar digital nerve in continuity with the mass [4].

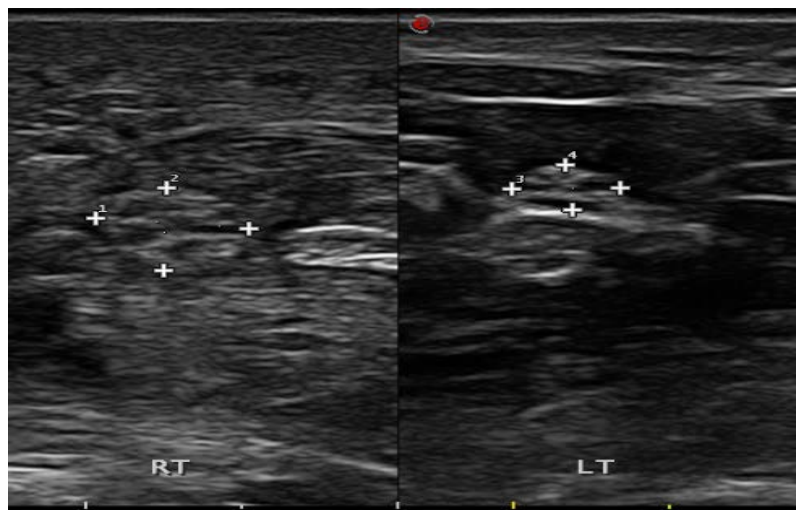

(a)

(b)

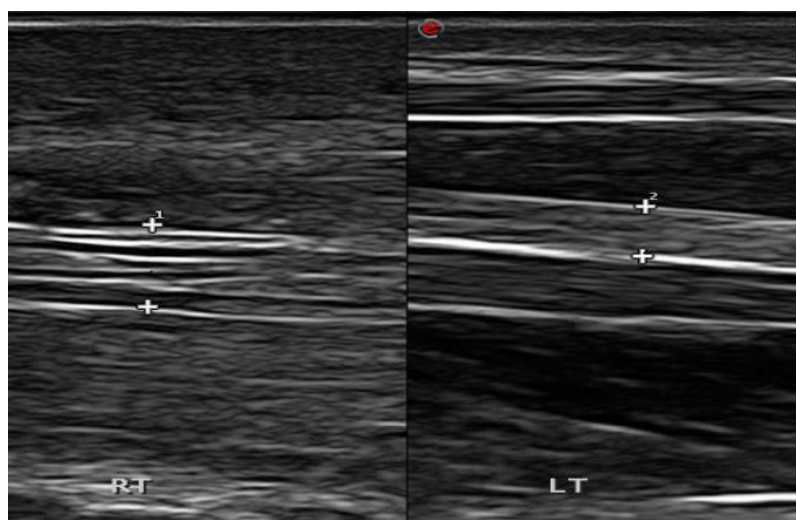

(c)

(d)

Figure 1. HRUS of median nerve in forearm in a 25-year-old gentle man with history of residual weakness three weeks after neurotmesis median nerve and status post nerve repair showed thickened edematous nerve.

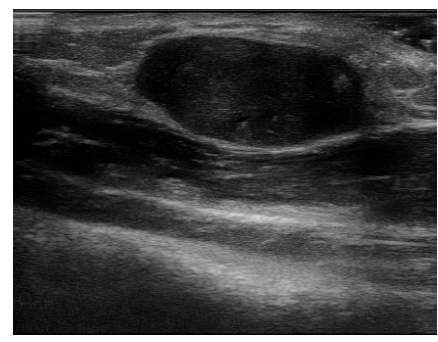

(a)

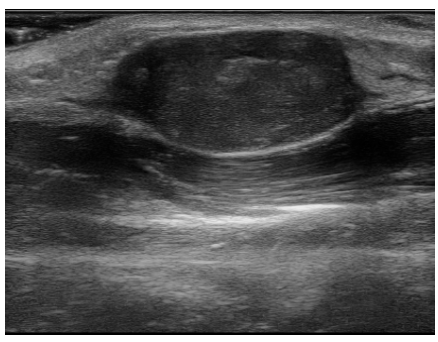

(b) 


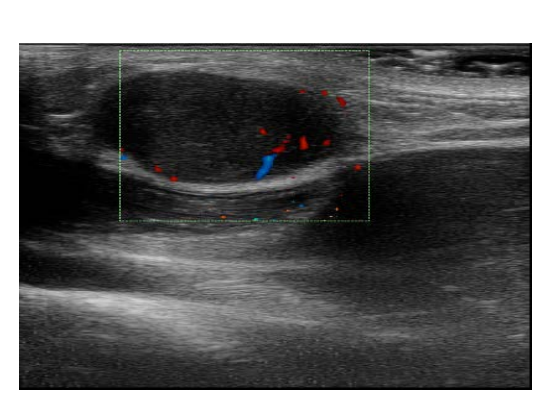

(c)

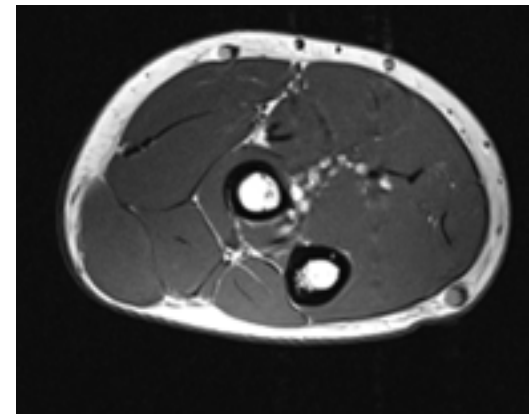

(e)

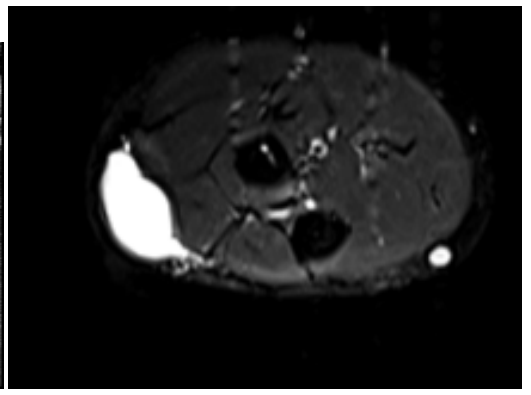

(d)

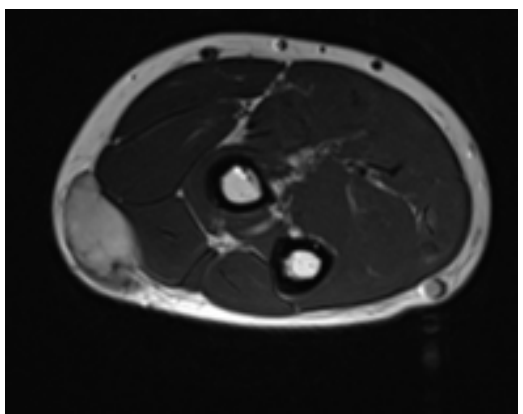

(f)

Figure 2. HRUS forearm in a 25 -year-old male patient with history of swelling since 4 months gradually increasing in size in forearm showed well defind oval shaped hypoechoic lesion with hyperechoic areas within, posterior acoustic enhancement and increased internal vascularity in subcutaneous plane. Lesion is in continuous with posterior cutaneous nerve of forearm with clearly defined nerve-tumor transition. MRI forearm showed well defined oval shaped lesion in subcutaneous plane, hypointense on T1W, hyperintense on T2W sequences and hyperintense on STIR images.

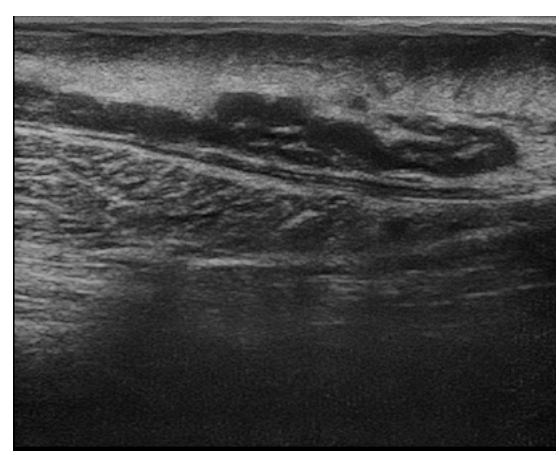

(a)

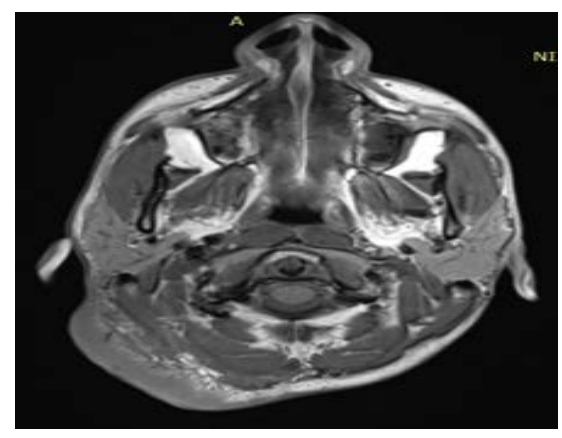

(c)

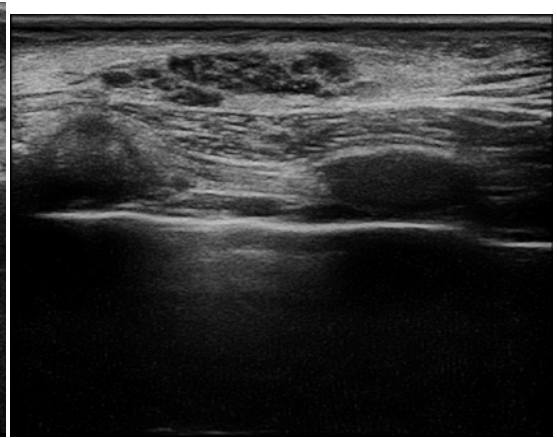

(b)

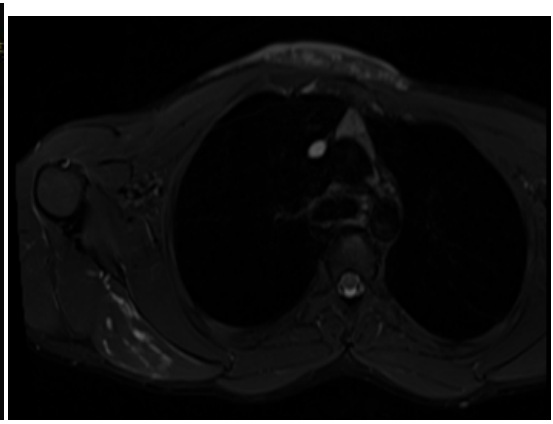

(d) 


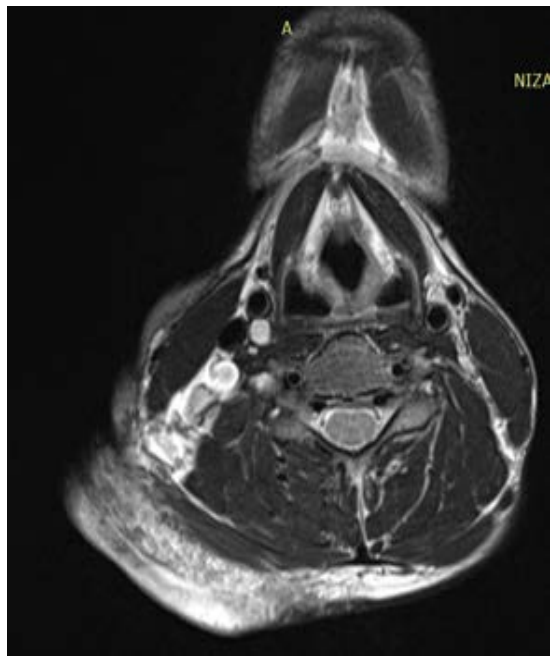

(e)

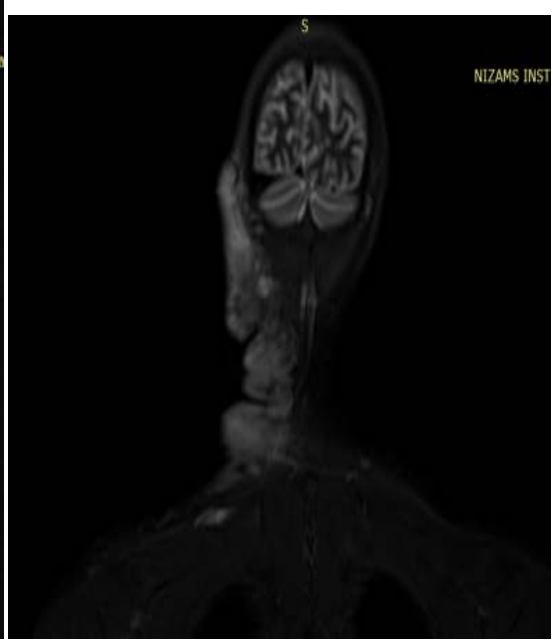

(f)

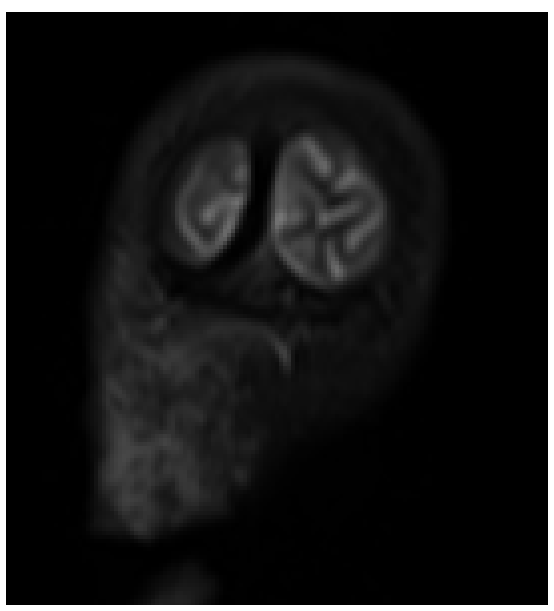

(g)

Figure 3. HRUS head and neck tumor in a 15-year-old boy with superficial mobile and multilobulated mass involving skin and subcutaneous fat head and neck on right side. The skin overlying this lesion is darkened with café au lait spots, suggestive of diffuse neurofibroma. MRI showed diffuse mass like lesion hypointense on T1-weighted, Hyperintense with central hypointense dots on T2 weighted sequences and tangle of hyperintense tubular cystic structures with central low signal on STIR imageswith target appearance. USG showed ill defined noncompressable heterogenous hypoechoic lesion consists of tangle of hypoechoic tubular structures lesion shows multiple arterial feeders.

\subsection{Lipofibromatous Hamartoma}

Lipofibromatous hamartoma is rare, slow growing, benign tumour like condition. Most commonly occurs in the peripheral nerves of the upper extremity with predilection for median nerve distally in the forearm and in its digital branches [11] [12]. Fibrolipomatous hamartoma may be associated with macrodystrophia lipomatosa-gigantism confined to the affected nerve territory [12] [13] produced by mesenchymal overgrowth confined to the affected nerve territory [Figure 4 and Figure 5]. There may be associated bone overgrowth causing macrodactyly [14]. 


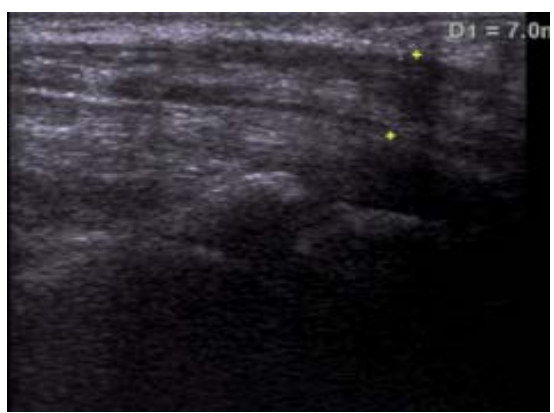

(a)

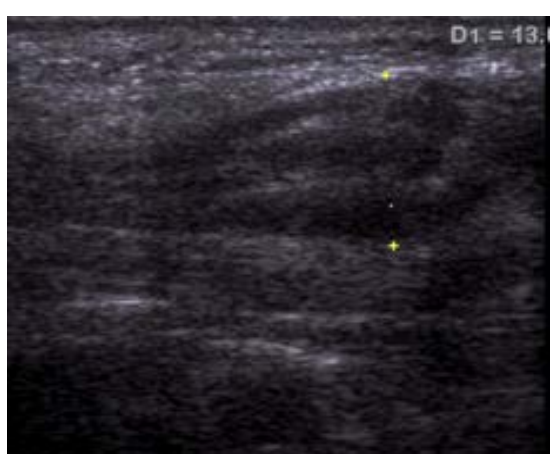

(c)

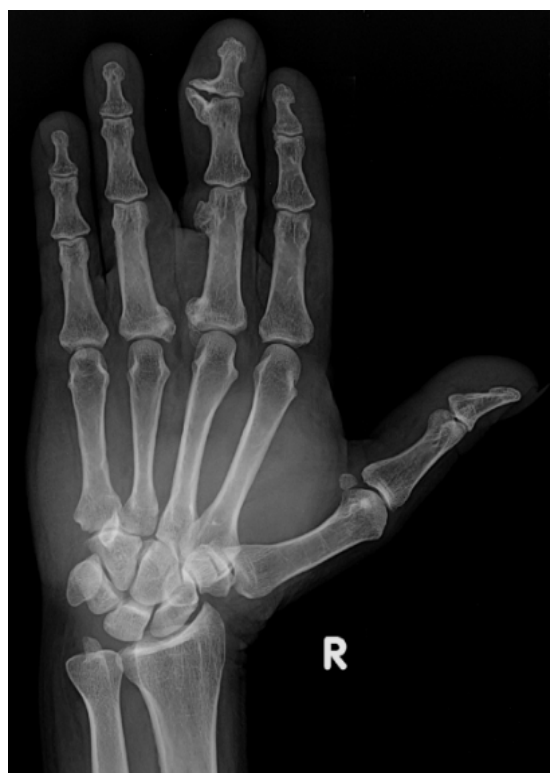

(e)

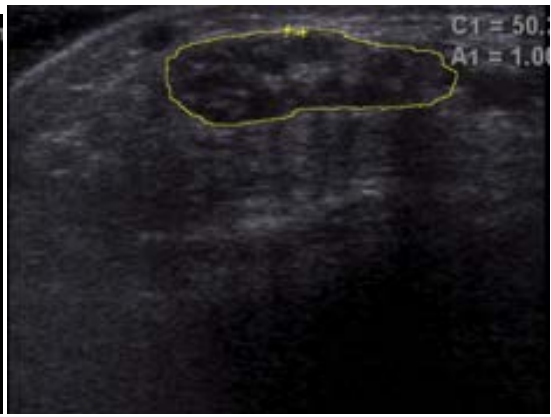

(b)

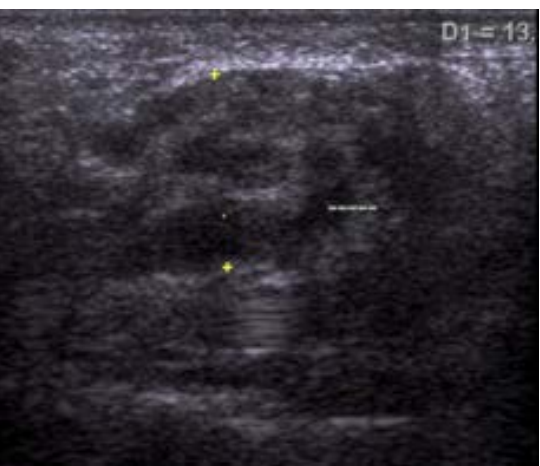

(d)

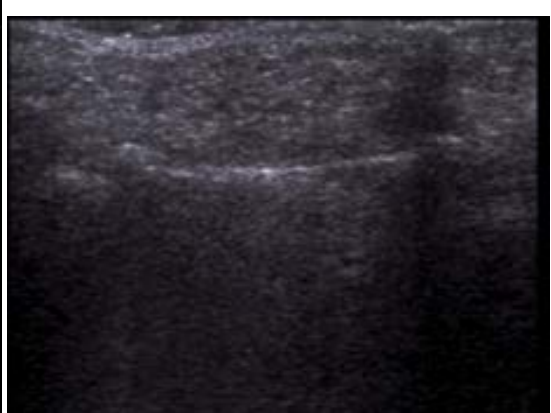

(f)

Figure 4. HRUS of right hand and wrist of 55-year-old gentleman with fibro lipomatous hamartoma of the median nerve about right wrist and hand with macrodystrophialipomatosa-gigantism showed enlargement of median nerve with hypoechoic linear serpentine fibers dispersed within hyperechoic substance suggestive of fatty infiltration, giving the appearance of "coaxial-cable-fibers" in the axial image (Figure 4(b) and Figure 4(d)) and serpiginous appearance on longitudinal images (Figure 4(a) and Figure 4(c)). Increased thickness of subcutaneous plane in the middle and ring fingers (Figure 4(f)). Radiograph of hand with wrist showed regional osseous gigantism in median nerve distribution, and premature osteoarthrosis of interphalyngealjoints (Figure 4(e)). 


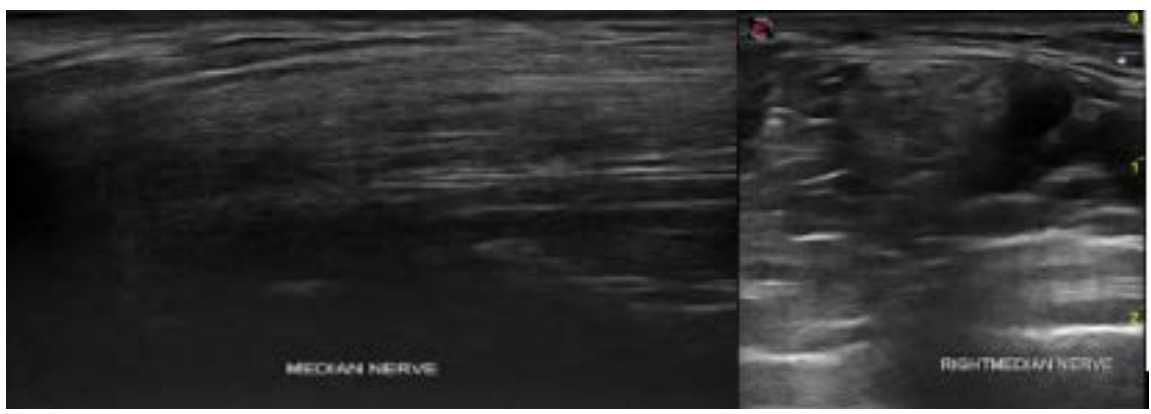

(a)

(b)

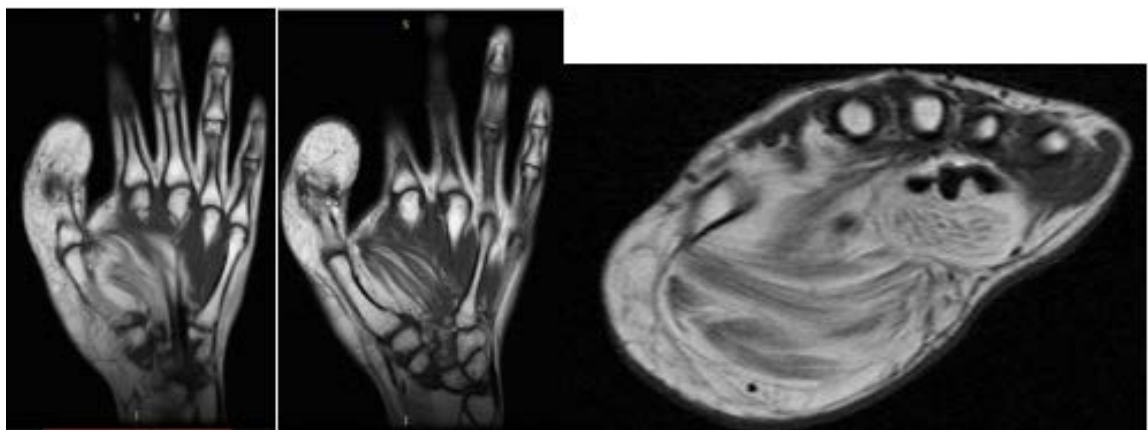

(c)

(d)

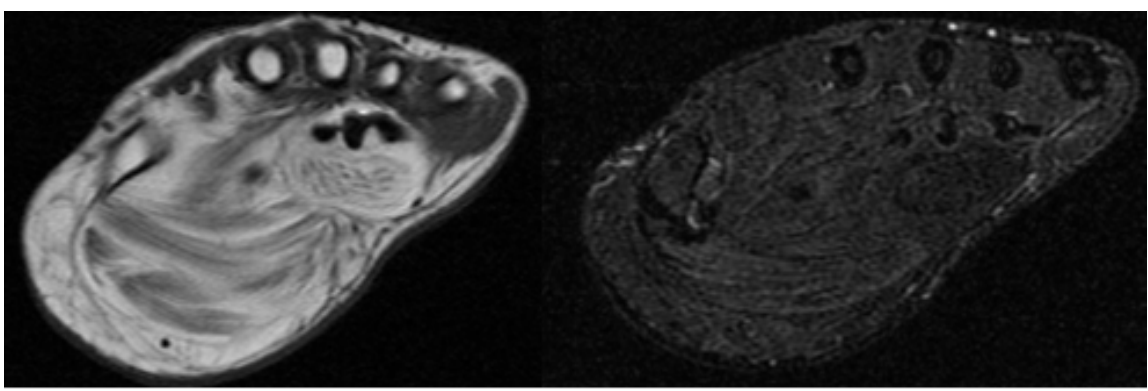

(e)

(f)

Figure 5. HRUS of hand and wrist in a 25-year-old female with diagnosis of fibro lipomatous hamartoma of the median nerve with macrodystrophia lipomatosa-gigantism showed enlargement of median nerve with hypoechoic linear serpentine fibers dispersed within hyperechoic substance suggestive of fatty infiltration, giving the appearance of "coaxial-cable-fibers" in the axial image and serpiginous appearance on longitudinal images. MRI of hand and wrist showed enlargement of median nerve with intermediate signal intensity linear serpentine fibers dispersed within hyperintense substance with T1W sequence, hypointense linear serpentine fibers dispersed within hyperintense substance with T2W sequence, STIR images showed complete suppression of hyperintense substance suggestive of fatty infiltration, giving the appearance of "coaxial-cable-fibers" in the axial image (Figure 5(d) and Figure 5(e)) and spaghetti like appearance on coronal images. Also there was increased thickness of soft tissues in thenar eminence, mid palm and in thumb with suggestion of hypertrophied subcutaneous fat and fatty infiltration of muscles (Figures 5(c)-(f)) and regional osseous gigantism thumb with premature osteoarthrosis of interphalyngeal joints.

Usually patients present with longstanding painless mass. Compression of the affected nerve with paresthesia, motor deficit and pain are known late symptoms [11] [13]. But compressive symptoms may occur only in median nerve involve- 
ment due to encroachment by the flexor retinaculum [11].

Macrodystrophia lipomatosa (MDL) is a rare disease typically causing localized gigantism and is often associated with a fibrolipomatosa hamartoma (FH) of the corresponding peripheral nerve. We present a case of carpal tunnel syndrome in an elderly man caused by fibrolipoma hamartoma of median nerve with associated macrodystrophia lipomatosa-gigantism confined to the median nerve territory [Figure 4 and Figure 5].

Imaging modalities used to diagnose the lesion are USG and MRI. USG and MRI characteristics reflect the histology of tumour [15].

APPEARANCE WITH USG: Involved nerve segment is enlarged (due to localized hypertrophy of epineural and perineural adipose and fibrous tissue) with thickened axonal bundles (due to endoneural and perineural fibrosis) dispersed within hypertrophied fat giving sausage shape [14] [16]. Pathognomonic ultrasonograhic characteristics are enlarged hyperechoic nerve containing hypertrophied fat and discrete hypoechoic cable like thickened axonal bundles giving coaxial cable like appearance on axial images and serpiginous appearance on longitudinal images [15] [16] [Figures 4-6].

Pathognomonic MR imaging characteristics are low signal intensity cylindrical bands (axonal bundles) surrounded by high signal intensity fat giving spaghetti like appearance on coronal planes and coaxial cable like appearance on axial planes on T1-weighted images [11] [13] [15]. Low signal intensity nerve fascicles are surrounded low signal intensity on fat-suppressed T2-weigted images [11] [Figure 5 and Figure 6].

\subsection{Infective Lesions}

In India, leprosy is a common treatable condition whose hallmark is nerve enlargement and inflammation. Clinical examination in leprosy may be subjective and inaccurate. Also, many nerves may not be amenable to palpation. Early detection of nerve impairment can help in preventing disability. USG provides subjective evidence of nerve enlargement and internal architecture. In leprosy, the nerves show enlargement and exhibit varying degrees of structural abnormalities such as edema, loss of fascicular architecture, increased nerve vascularity on doppler with or without central necrosis and nerve abscess [5] [Figure 7].

\subsection{Entrapment Neuropathies}

The nerves are more prone to compression in specific locations where they course through osteofibrous tunnels [1]. The median nerve in carpal tunnel and ulnar nerve in Guyon's canal and cubital tunnel are the common sites of entrapment in the upper limb. Common peroneal nerve near fibular neck and posterior tibial nerve in tarsal tunnel are commonly involved in the lower limb. Hallmark of nerve entrapment is thickened and hypoechoic nerve segment at and proximal to the level of entrapment. The most consistent finding in HRUS for CTS is a significant increase in the cross-sectional area (CSA) at the pisiform 
bone level (equivalent to the carpal tunnel inlet). The best discriminatory criterion for USG diagnosis of carpal tunnel syndrome are median nerve cross sectional area in the proximal part of carpal tunnel $\geq 9 \mathrm{~mm}^{2}$ [4] [17] [Figure 8].

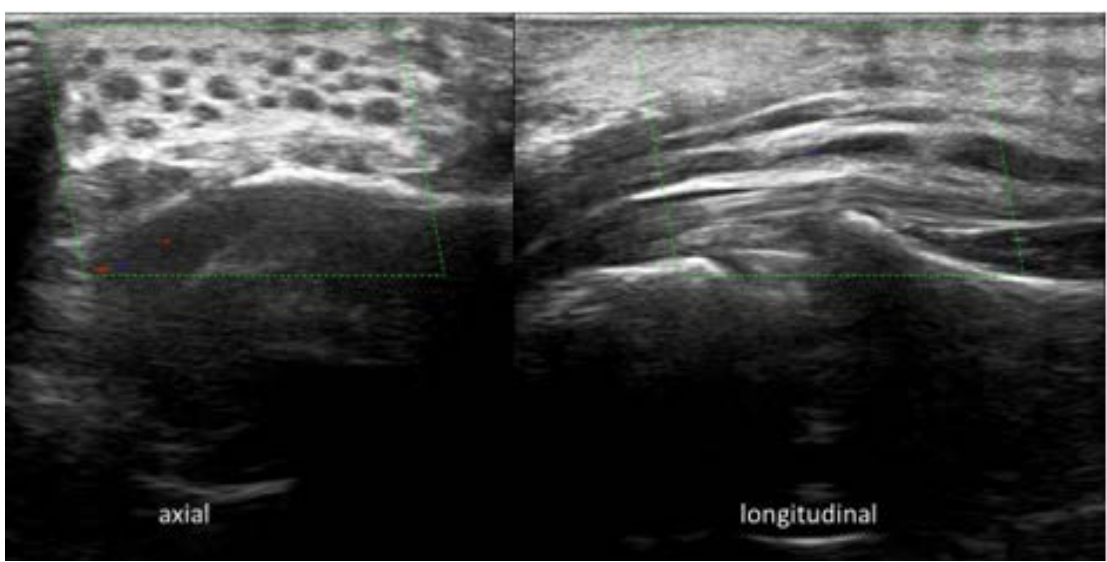

(a)

(b)

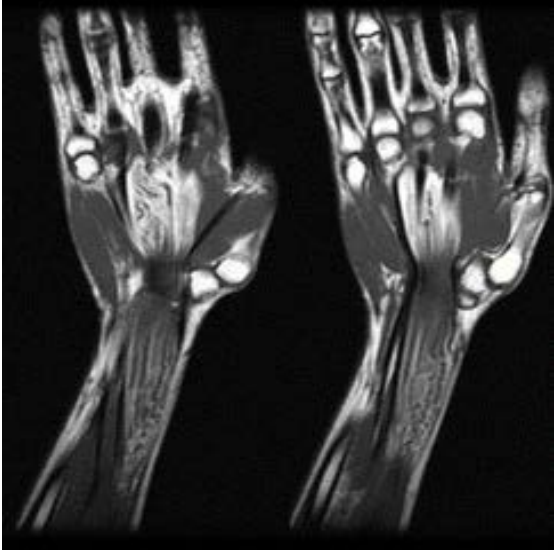

(c)

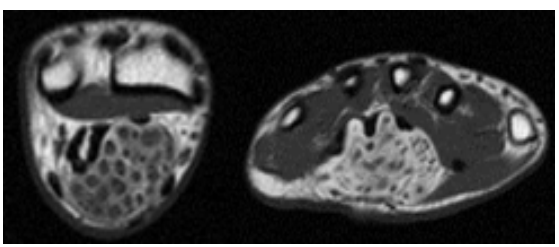

(e)

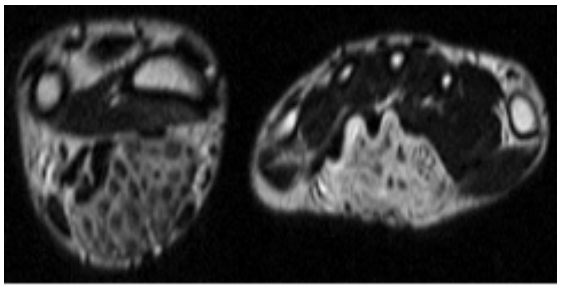

(d)

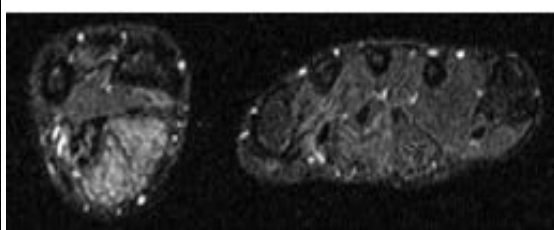

(f)

Figure 6. HRUS of hand and wrist of 19-year-old gentleman with fibro lipomatous hamartoma of the median nerve showed enlargement of median nerve with hypoechoic linear serpentine fibers dispersed within hyperechoic substance suggestive of fatty infiltration, giving the appearance of "coaxial-cable-fibers" in the axial image (Figure 6(a)) and serpiginous appearance on longitudinal images (Figure 6(b)). MRI of hand and wrist showed enlargement of median nerve with intermediate signal intensity linear serpentine fibers dispersed within hyperintense substance with T1W sequence, hypointense linear serpentine fibers dispersed within hyperintense substance with T2W sequence, STIR images showed complete suppression of hyperintense substance suggestive of fatty infiltration (Figure 6(f)), giving the appearance of "coaxial-cable-fibers" in the axial image (Figure 6(d) and Figure 6(e)) and spaghetti like appearance on coronal images (Figure 6(c)). 


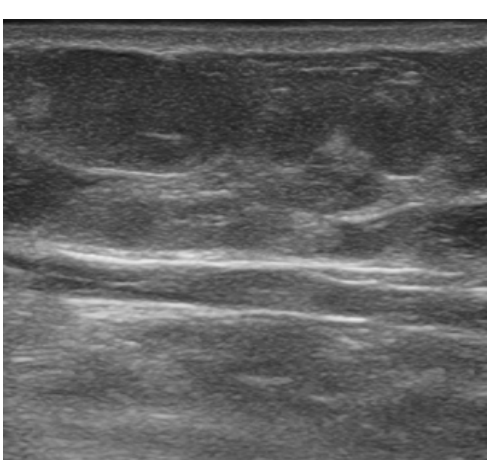

(a)

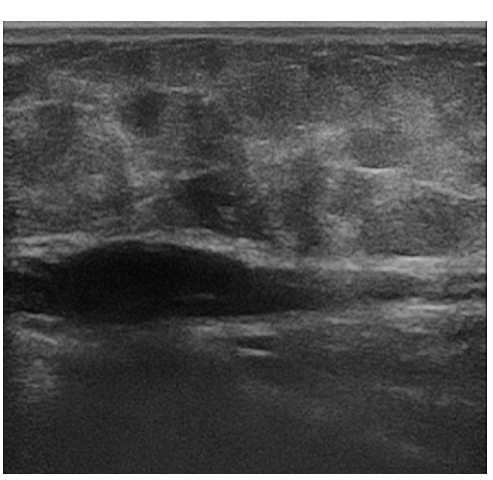

(c)

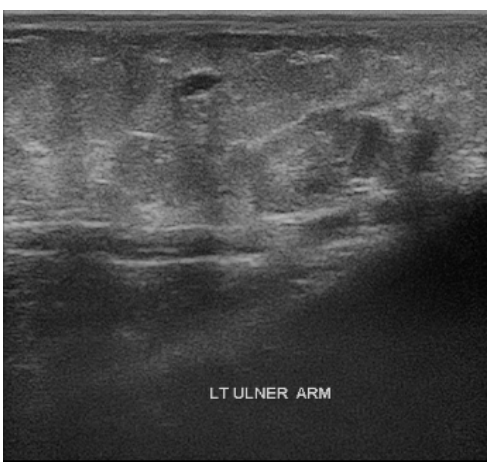

(e)

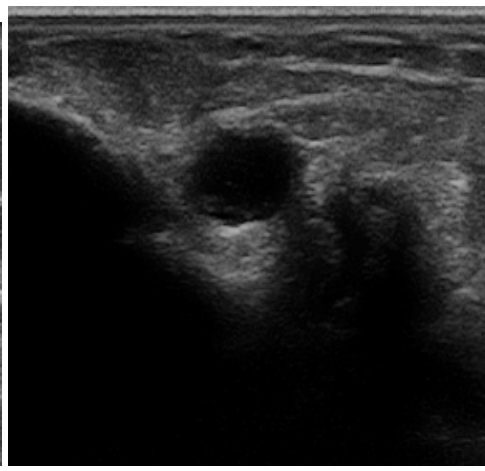

(b)

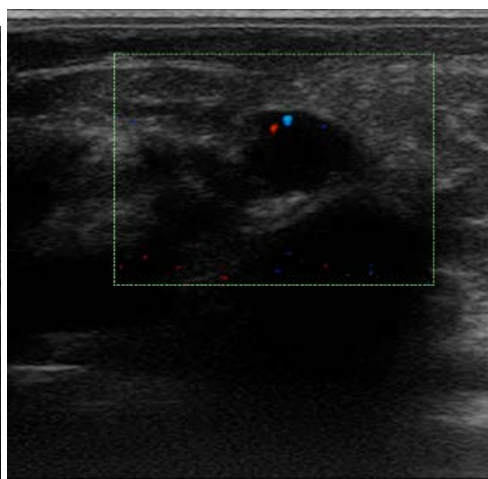

(d)

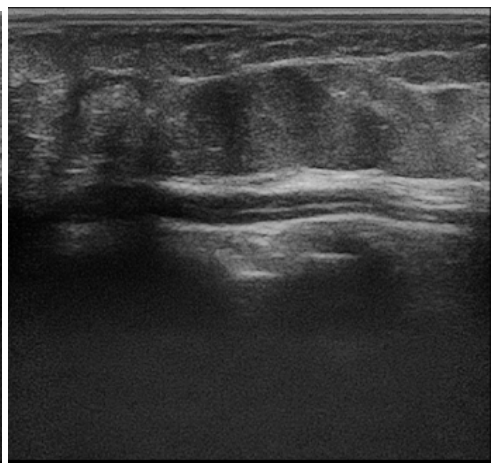

(f)

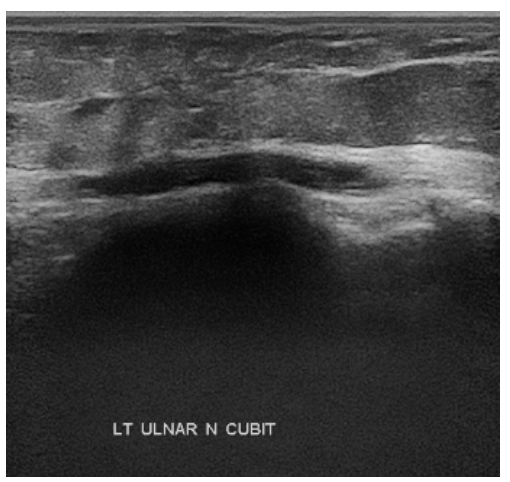

(g)

Figure 7. HRUS both ulnar nerves in cubital tunnel in a 45-year-old female suspected to have hansen's disease showed well defined anechoic cystic lesions in both the nerves suggestive of nerve abscess. 


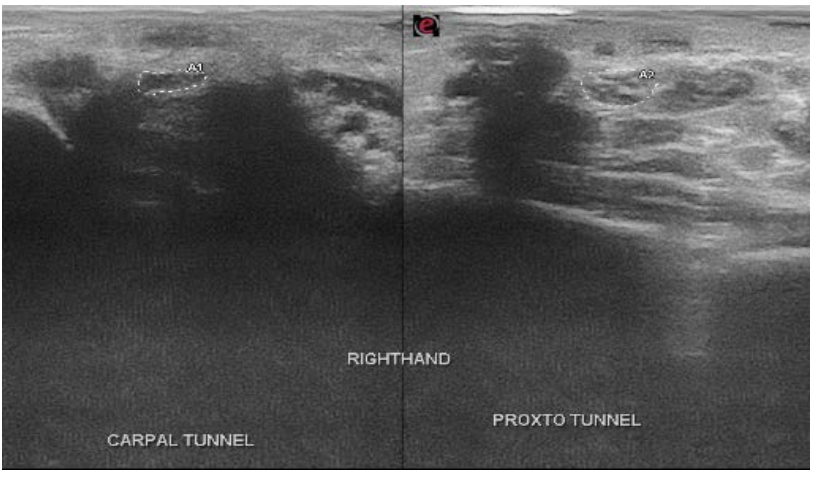

(a)

(b)

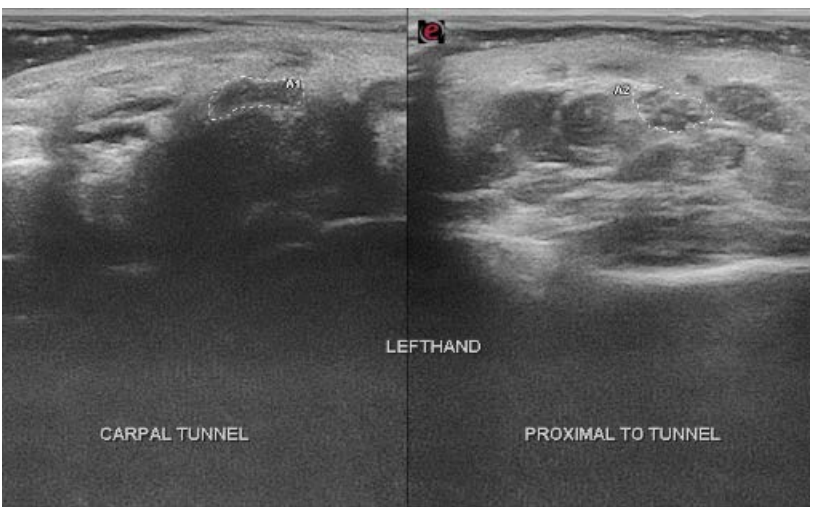

(c)

(d)

Figure 8. HRUS of right wrist in a 67-year-old gentle man with history of status post carpal tunnel decompression with persistent carpal tunnel syndrome showed diffuse thickening of right median nerve with statistically significant flattening of intracarpal segment and ganglion cyst in wrist joint. Screening HRUS of left median and both the ulnar nerves as well revealed thickened peripheral nerves suggestive of peripheral neuropathy.

\section{Various Nerve Lesions Diagnosed with HRUS}

CASE 1: A 55-year-old gentleman presented with weakness and progressively increasing painless swelling in the volar aspect of right wrist and palm for 20 years. On examination there was swelling of the thenar eminence, middle and index fingers. High resolution ultrasonography of hand and wrist showed enlargement of median nerve with hypoechoic linear serpentine fibers dispersed within hyperechoic substance suggestive of fatty infiltration, giving the appearance of "coaxial-cable-fibers" in the axial image and serpiginous appearance on longitudinal images. Nerve thickening was extending proximally within the carpal tunnel and distally involving the digital branches. The mass caused bulging of the flexor retinaculum. Also there was increased thickness of subcutaneous plane in the middle and ring fingers. Radiograph of hand with wrist showed regional osseous gigantism in median nerve distribution, and premature osteoarthrosis of interphalyngeal joints.

A diagnosis of fibro lipomatous hamartoma of the median nerve with macrodystrophia lipomatosa-gigantism causing carpal tunnel syndrome was made. 
Nerve conduction studies revealed reduction in median nerve conduction at wrist and hand.

CASE 2: A 19-year-old gentleman presented with painless swelling at wrist present since birth, gradually increasing in size with no sensory or motor deficits. On examination there was swelling of the wrist. MRI of hand and wrist showed enlargement of median nerve with intermediate signal intensity linear serpentine fibers dispersed within hyperintense substance with T1W sequence, hypointense linear serpentine fibers dispersed within hyperintense substance with T2W sequence, STIR images showed complete suppression of hyperintense substance suggestive of fatty infiltration, giving the appearance of "coaxial-cable-fibers" in the axial image and spaghetti like appearance on coronal images. Nerve thickening was extending proximally into the forearm and distally involving the digital branches.

High resolution ultrasonography of hand and wrist showed enlargement of median nerve with hypoechoic linear serpentine fibers dispersed within hyperechoic substance suggestive of fatty infiltration, giving the appearance of "coaxial-cable-fibers" in the axial image and serpiginous appearance on longitudinal images. Nerve thickening was extending proximally into the forearm and distally involving the digital branches. The mass caused bulging of the flexor retinaculum.

A diagnosis of fibro lipomatous hamartoma of the median nerve was made.

CASE 3: A 25-year-old female patient presented with hypertrophied thenar eminence and thumb since birth and pain in wrist for 6 months. MRI of hand and wrist showed enlargement of median nerve with intermediate signal intensity linear serpentine fibers dispersed within hyperintense substance with T1W sequence, hypointense linear serpentine fibers dispersed within hyperintense substance with T2W sequence, STIR images showed complete suppression of hyperintense substance suggestive of fatty infiltration, giving the appearance of "coaxial-cable-fibers" in the axial image and spaghetti like appearance on coronal images. Nerve thickening was extending proximally into the forearm and distally involving the digital branches.

Also there was increased thickness of soft tissues in thenar eminence, mid palm and in thumb with suggestion of hypertrophied subcutaneous fat and fatty infiltration of muscles and regional osseous gigantism thumb with premature osteoarthrosis of interphalyngeal joints.

High resolution ultrasonography of hand and wrist showed enlargement of median nerve with hypoechoic linear serpentine fibers dispersed within hyperechoic substance suggestive of fatty infiltration, giving the appearance of "coaxial-cable-fibers" in the axial image and serpiginous appearance on longitudinal images. Nerve thickening was extending proximally into the forearm and distally involving the digital branches. The mass caused bulging of the flexor retinaculum.

A diagnosis of fibro lipomatous hamartoma of the median nerve with macrodystrophia lipomatosa-gigantism causing carpal tunnel syndrome was made. 
CASE 4: A 45-year-old female patient with history of vague symptoms like generalised weakness, malaise, mild joint pain, clinically suspected to be hansen's disease. HRUS both ulnar nerves in cubital tunnel showed well defined anechoic cystic lesions in both the nerves suggestive of nerve abscess.

CASE 5: A 67-year-old gentle man with history of pain in right wrist joint and weakness in hand diagnosed to have very tight carpal tunnel with gross compression of median nerve of right hand with HRUS and ENMG in July 2017. Patient underwent carpal tunnel decompression on right hand in Aug 2017. Even after decompression there was no improvement in pain and weakness. Again ENMG was done in Nov 2017, which showed no change in electrophysiology of median nerve compared to pre decompression ENMG which was suggestive of bilateral carpal tunnel syndrome (Right > Left). HRUS done in Nov 2017 showed diffuse thickening of peripheral nerves suggestive peripheral neuropathy and ganglion cyst in right wrist joint. Right median nerve measured $0.19 \mathrm{~cm}^{2}$ proximal to carpal tunnel and $0.10 \mathrm{~cm}^{2}$ in the carpal tunnel with statistically significant flattening of intracarpal segment in keeping with clinical diagnosis of carpal tunnel syndrome. Left median nerve enlarged in its entire course, measured 0.19 $\mathrm{cm}^{2}$ proximal to tunnel and $0.20 \mathrm{~cm}^{2}$ within the tunnel with no suggestion of carpal tunnel syndrome either clinically or with imaging. Screening HRUS of both the ulnar nerves revealed thickened ulnar nerves as well suggestive of peripheral neuropathy.

CASE 6: A 25-year-old gentle man met with an accident and had crush injury right forearm, brought to hospital with deficits in median nerve distribution of hand. After complete evaluation found to have neurotmesis median nerve and nerve repair was done. Three weeks after repair patient still continued to have residual weakness. USG showed thickened edematous nerve.

Case 7: A 25-year-old gentle man research scholar by occupation came with history of pain less swelling forearm, gradually progressing in size since 4 months. HRUS forearm forearm showed well defind oval shaped hypoechoic lesion with hyperechoic areas within, posterior acoustic enhancement and increased internal vascularity in subcutaneous plane. Lesion is in continuous with posterior cutaneous nerve of forearm with clearly defined nerve-tumor transition. MRI forearm showed welldefined oval shaped lesion in subcutaneous plane, hypointense on T1W, hyperintense on T2W sequences and hyperintense on STIR images.

CASE 8: A 15-year-old boy with superficial, multilobulated, mobile mass involving skin and subcutaneous fat head and neck on right side since birth, gradually increasing in size. The skin overlying this lesion is darkened with café au lait spots, suggestive of diffuse neurofibroma. MRI showed diffuse mass like lesion hypointense on T1-weighted, Hyperintense with central hypointense dots on T2 weighted sequences, tangle of hyperintense tubular structures with central low signal intensity with target appearance on STIR images. USG showed ill defined noncompressable heterogenous hypoechoic lesion consists of tangle of 
hypoechoic tubular structures. Lesion shows multiple arterial feeders.

\section{Conclusion}

Ultrasonography is an excellent imaging tool owing to its ability to scan entire course of nerve quickly and efficiently, is cost-effective, can diagnose and localize various pathologies that involve the peripheral nerves such as traumatic, inflammatory, infective, neoplastic, and traumatic neuropathies. USG exceptionally has an important role to play in evaluation of patients with suspected nerve injury, as MRI may not differentiate neural contusion from nerve disruption.

\section{Conflicts of Interest}

The authors declare no conflicts of interest regarding the publication of this paper.

\section{References}

[1] Brown, J.M., Yablon, C.M., Morag, Y., Brandon, C.J. and Jacobson, J.A. (2016) US of the Peripheral Nerves of the Upper Extremity: A Landmark Approach. RadioGraphics, 36, 452-463. https://doi.org/10.1148/rg.2016150088

[2] Suk, J.I., Walker, F.O. and Cartwright, M.S. (2013) Ultrasound of Peripheral Nerves. Current Neurology and Neuroscience Reports, 13, 328. https://doi.org/10.1007/s11910-012-0328-x

[3] Koenig, R.W., Pedro, M.T., Heinen ,C.P., Schmidt, T., Richter, H.P., Antoniadis, G. and Kretschmer, T. (2009) High-Resolution Ultrasonography in Evaluating Peripheral Nerve Entrapment and Trauma. Neurosurgical Focus, 26, E13. https://doi.org/10.3171/FOC.2009.26.2.E13

[4] Jacobson, J.A., Wilson, T.J. and Yang, L.J. (2016) Sonography of Common Peripheral Nerve Disorders with Clinical Correlation. Journal of Ultrasound Medicine, 35, 683-693. https://doi.org/10.7863/ultra.15.05061

[5] Kharat, A., Thakkar, D.K., Jantre, M. and Singh, A. (2014) Role of High-Resolution Ultrasound in Diagnosis and Follow-Up of Leprosy Patients. Medical Journal of Dr D.Y. Patil Vidyapeeth, 7, 686.

[6] Bohm, J., Scheidl, E., Bereczki, D., Schelle, T., Aranyi, Z. (2014) High Resolution Ultrasonography of Peripheral Nerves: Measurements on 14 Nerve Segments in 56 Healthy Subjects and Reliability Assessments. Ultraschall in der Medizin, 35, 459-467. https://doi.org/10.1055/s-0033-1356385

[7] Congress of Neurological Surgeons (2017) Diagnosis and Management of Peripheral Nerve Injury and Entrapment.

[8] Ryu J.A.H., Lee, S.H., Cha, E.-Y., Kim, T.Y., Kim, S.M. and Shin, M.J. (2015) Sonographic Differentiation between Schwannomas and Neurofibroma in the Musculoskeletal System. Journal of Ultrasound in Medicine, 34, 2253-2260.

https://doi.org/10.7863/ultra.15.01067

[9] Lee, S.J. and Yoon, S.T. (2017) Ultrasonographic and Clinical Characteristics of Schwannoma of the Hand. Clinics in Orthopedic Surgery, 9, 91-95. https://doi.org/10.4055/cios.2017.9.1.91

[10] Lim, R., Jaramillo, D., Poussaint, T.Y. and Chang, Y. (2005) Superficial Neurofibroma: A Lesion with Unique MRI Characteristics in Patients with Neurofibroma- 
tosis Type1. American Journal of Roentgenology, 184, 962-968. https://doi.org/10.2214/ajr.184.3.01840962

[11] Al-Jabri, T., Garg, S. and Mani, G.V. (2010) Lipofibromatous Hamartoma of the Median Nerve. Journal of Orthopaedic Surgery and Research, 5, 71.

[12] Meyer, B.-U. and Röricht, S. (1997) Fibrolipomatous Hamartoma of the Proximal Ulnar Nerve Associated with Macrodactyly and Macrodystrophia Lipomatosa as an Unusual Cause of Cubital Tunnel Syndrome. Journal of Neurology, Neurosurgery and Psychiatry, 63, 808-810. https://doi.org/10.1136/jnnp.63.6.808a

[13] Nilson, J., Sandberg, K., Dahlin, L.B., Vendel, N., Balslev, E., Larsen, L. and Nielsen, N.S. (2010) Fibrolipomatous Hamartoma in the Median Nerve in the Arm-An Unusual Location But with MR Imaging Characteristics: A Case Report. Journal of Brachial Plexus and Peripheral Nerve Injury, 5, 1.

[14] Ha, J.F., The, B.M., Abeysuriya, D.T.D. and Luo, D.Y.W. (2012) Fibrolipomatous Hamartoma of the Median Nerve in the Elbow: A Case Report. Summer, 12, 152-154.

[15] Plaza, J.A., Kazakov, D.V., Cases, G., et al. (2014) Fibrolipomatous Hamartoma of the Nerve: A Clinicopathologic Report of 13 Cases. Journal of the American Academy of Dermatology, 70, 736-742. https://doi.org/10.1016/j.jaad.2013.10.002

[16] Louaste, J., Zejjari, H., Chkoura, M., Houmadi, A. and Rachid, K. (2011) Carpal Tunnel Syndrome Due to Fibrolipomatous Hamartoma of the Median Nerve. Hand (NY), 6, 76-79. https://doi.org/10.1007/s11552-010-9290-8

[17] Kamath, J.B., Reddy, B., Sivam, U., Venugopal, A. and Jayasheelan, N. (2016) Carpal Tunnel Syndrome: Ultrasonographic Evaluation of Median Nerve Diameter. Journal of Orthopedics and Allied Sciences, 4, 14-17.

https://doi.org/10.4103/2319-2585.180690 